\title{
Simultaneous Particle Tracking and Temperature Measurements during Additive Manufacturing using a High-speed Spectral Plenoptic Camera
}

\author{
Dustin Kelly ${ }^{1}$, Ralf Fischer ${ }^{2}$, Ari Goldman ${ }^{1}$, Sarah Morris ${ }^{1}$, Bart Prorok ${ }^{2}$, and \\ Brian Thurow ${ }^{1}$ \\ ${ }^{1}$ Auburn University, Advanced Flow Diagnostics Laboratory, Auburn, AL USA \\ 2 Auburn University, Materials Research and Education Center, Auburn, AL USA
}

In this work, a high-speed spectral plenoptic camera was used for three-dimensional (3D) simultaneous particle tracking and pyrometry measurements of hot spatter particles ejected during the metal additive manufacturing process. Additive manufacturing (AM) has an increasing role in the aerospace, energy, medical and automotive industry (DebRoy et al., 2018). While this new technology enables the production of highly advanced parts, research on the fundamental mechanisms governing the laser-matter interactions are an ongoing challenge because of the spatial and temporal resolution inherent to the AM process. One challenge is the characterization of spatter particles ejected from the melt pool, as these particles can be incorporated into the final part affecting the mechanical properties (Deng et al., 2020). One potential solution for simultaneously measuring velocity and temperature of the spatter particles is the spectral plenoptic camera.

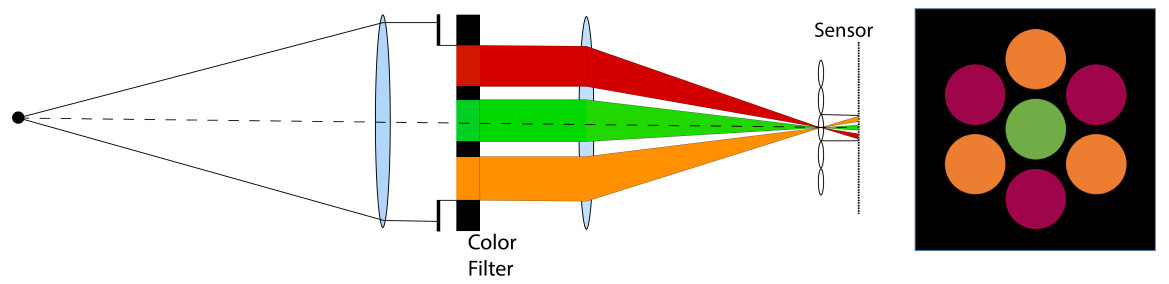

Figure 1: (Left) A simple schematic of a three color spectral plenoptic camera showing how a single microlens captures an image of the aperture plan, and (right) the band pass filter array layout for these experiments where $800 \mathrm{~nm}$ is red, $600 \mathrm{~nm}$ is orange, and $532 \mathrm{~nm}$ is green.

The plenoptic camera is a type of light-field camera, which provides the ability to instantaneously capture both angular and spatial information with one sensor. Adelson and Wang (1992) developed the modern design of a plenoptic camera, which is composed of a microlens array placed in front of a relay lens that images the microlens array onto a traditional sensor. Each microlens of the array images the aperture plane, where each pixel discretizes the aperture, capturing the angular information as shown in Figure 1 . A convenient way to parameterize plenoptic images is through two-plane parameterization as described by Levoy (2006), where the u-v and s-t planes correspond to the aperture and microlens planes, respectively. Plenoptic imaging provides the ability to generate perspective images of the scene by adjusting the $(\mathrm{u}, \mathrm{v})$ coordinate. A recent modification of the plenoptic camera, first introduced by Danehy et al. (2017), adds spectral filters at the aperture plane of the main lens. Each microlens captures sub-images of the spectral filters as shown in Figure 1. The spectral plenoptic camera provides the ability to individually select spectral filters, where each filter possesses limited angular information. Increasing the number of different spectral filters decreases the overall angular information for a particular wavelength/spectrum.

The current implementation of the spectral module and the high-speed plenoptic camera has a maximum of seven filters as shown in Figure 1 , in which there were three different spectral $10 \mathrm{~nm}$ band-pass filters as indicated by the filter layout image. The camera used was a Phantom VEO4k high-speed camera capable of $1000 \mathrm{~Hz}$ at a resolution of $(2048 \times 2048)$ with a microlens array $(471 \times 362)$ placed in front of a relay lens that relays the microlens images to the sensor. The spectral module is composed of two lenses designed for a working distance/magnification and to f-match the microlens array as described by Fahringer et al. (2018), where a magnification of 0.6 was chosen for these experiments. 
The first step of the PTV algorithm is locating the particles in the volume for each frame using plenoptic ray bundling, first described by Clifford et al. (2019). Ray bundling takes advantage of the two plane parameterization, where perspective images are first generated and particle locations are found through applying a minimum threshold. The perspective is the $(\mathrm{u}, \mathrm{v})$ coordinate and the location on the image is the $(\mathrm{s}, \mathrm{t})$ coordinate of the rays found during thresholding. Every ray is then traced between the two planes, where the particle locations correspond to the point where the rays of different perspectives converge. This corresponds to a 3D location in the image space, which is converted to the object space. Velocity measurements were made using a central finite time difference particle tracking technique, wherein particles' 3D locations were matched in the images. Ray bundling was conducted with the (i) $600 \mathrm{~nm}$ filter perspectives, (ii) 800 $\mathrm{nm}$ filter perspectives, and (iii) all perspectives to determine the uncertainty.

Dual-wavelength pyrometry was used to estimate the particle temperatures, where temperature is determined by the ratio of the average intensity between $600 \mathrm{~nm}$ and $800 \mathrm{~nm}$ filters for each microlens $\left(r=I_{600 \mathrm{~nm}} / I_{800 \mathrm{~nm}}\right)$. Temperature measurements are limited to the focal plane of the camera due to the assumption that the spectral information captured by one microlens originates from one particle, which only occurs at the focal plane of the camera. Further improvements to the pyrometry algorithm could remove this limitation. The temperature calibration was performed in a Lindberg/Blue M tube furnace. An Inconel 625 shim with a thermocouple attached to it was placed in the center of the furnace and temperature calibration images were captured at temperatures ranging between $950^{\circ} \mathrm{C}$ and $1250^{\circ} \mathrm{C}$ at intervals of $50^{\circ} \mathrm{C}$.

The AM experiment was performed on a customized AM machine at Auburn University's Materials Research and Education Center. The laser source was an IPG YLR-400AC continuous wave ytterbium fiber laser with a wavelength of $1070 \mathrm{~nm}$ and a maximum output power of $400 \mathrm{~W}$. An IPG Mid-Power Scanner with a $250 \mathrm{~mm}$ f-theta lens was used to deliver the beam onto a 10x10 mm Inconel 625 substrate. An Inconel 625 powder layer of about $30 \mu \mathrm{m}$ was spread onto the substrate. The laser scan path was a single continuous line scan with $800 \mathrm{~mm} / \mathrm{s}$ scan velocity and $250 \mathrm{~W}$ laser power, and the laser traversed the $\mathrm{z}=0$ plane of the volume from right to left in Figure 2. The $8.5 \mathrm{~mm}$ long scan was performed below the scan head and perpendicular to the direction of view of the plenoptic camera. The single line scan was performed under ambient conditions for the demonstration of the capabilities of the plenoptic camera.

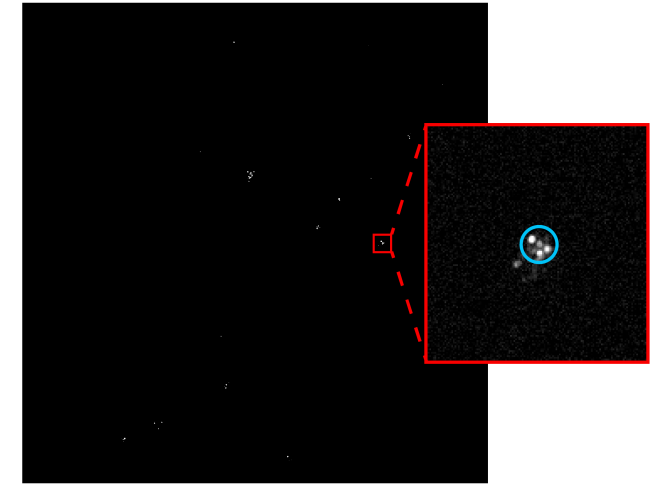

(a)

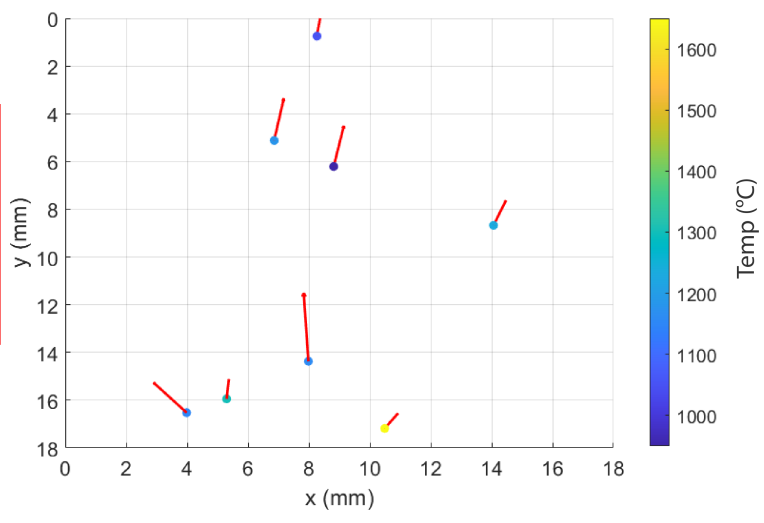

(b)

Figure 2: (a) Spectral plenoptic image with a zoomed image with a blue circle corresponding to a single microlens that images a particle, (b) particle locations with the color corresponding to temperature with velocity vectors $(\mathrm{mm} / \mathrm{ms})$.

Figure 2(a) shows a spectral plenoptic image of a high temperature particle field generated by the additive manufacturing process. Figure 2 b) shows particle locations along the $x-y$ plane calculated using ray bundling where the particle color corresponds to the temperature. The velocity vector size is scaled with velocity $(\mathrm{mm} / \mathrm{ms})$. Particle velocities ranged between $0.3 \mathrm{~m} / \mathrm{s}$ to $7 \mathrm{~m} / \mathrm{s}$, where the faster particles originated in the first $5 \mathrm{~ms}$, which agrees with the velocities found by Guo et al. (2018). Traditionally with single plenoptic reconstructions, the uncertainty in the z-direction is 6 to 10 times larger than in the $\mathrm{x}$ and $\mathrm{y}$ directions. This is on a similar order even with the reduced angular information per wavelength. The majority of particle temperatures ranged between $1000^{\circ} \mathrm{C}$ to $1600^{\circ} \mathrm{C}$. With a melting temperature of approximately $1300^{\circ} \mathrm{C}$, the Inconel 625 particles were present in both liquid and solid states. For particles along the focal plane, a general cooling trend of approximately $150^{\circ} \mathrm{C} / \mathrm{ms}$ was observed. The measurement uncertainty for both particle location and temperature are still a current topic of investigation. 


\section{Acknowledgements}

This work was sponsored by the United States National Institute of Standards and Technology under contracts NIST-70NANB16H272 and NIST-70NANB18H220. The authors gratefully acknowledge the support of Mr. Steven Moore and Mr. Emre Kayali at Auburn University's Materials Research and Education Center.

\section{References}

Adelson E and Wang J (1992) Single lens stereo with a plenoptic camera. IEEE Transactions on Pattern Analysis and Machine Intelligence 14:99-106

Clifford C, Tan Z, Hall E, and Thurow B (2019) Particle matching and triangulation using light-field ray bundling. 13th International Symposium on Particle Image Velocimetry

Danehy PM, Hutchins WD, Fahringer TW, and Thurow BS (2017) A Plenoptic Multi-Color Imaging Pyrometer. 55th AIAA Aerospace Sciences Meeting pages 1-7

DebRoy T, Wei H, Zuback J, Mukherjee T, Elmer J, Milewski J, Beese A, Wilson-Heid A, De A, and Zhang W (2018) Additive manufacturing of metallic components - process, structure and properties. Progress in Materials Science 92:112-224

Deng P, Karadge M, Rebak RB, Gupta VK, Prorok BC, and Lou X (2020) The origin and formation of oxygen inclusions in austenitic stainless steels manufactured by laser powder bed fusion. Additive Manufacturing 35:101334

Fahringer TW, Danehy PM, and Hutchins WD (2018) Design of a Multi-Color Plenoptic Camera for Snapshot Hyperspectral Imaging. 2018 Aerodynamic Measurement Technology and Ground Testing Conference pages $1-9$

Guo Q, Zhao C, Escano LI, Young Z, Xiong L, Fezzaa K, Everhart W, Brown B, Sun T, Chen L, and et al (2018) Transient dynamics of powder spattering in laser powder bed fusion additive manufacturing process revealed by in-situ high-speed high-energy x-ray imaging. Acta Materialia 151:169-180

Levoy M (2006) Light Fields and Computational Imaging. Computer 39:46-55 